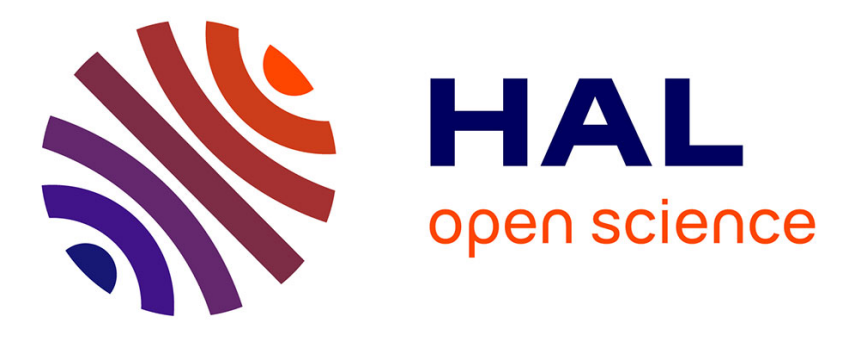

\title{
Memory Resilient Gain-scheduled State-Feedback Control of LPV Time-Delay Systems with Time-Varying Delays
}

Corentin Briat, Olivier Sename, Jean-François Lafay

\section{To cite this version:}

Corentin Briat, Olivier Sename, Jean-François Lafay. Memory Resilient Gain-scheduled StateFeedback Control of LPV Time-Delay Systems with Time-Varying Delays. ROCOND 2009 - 6th IFAC Symposium on Robust Control Design, Jun 2009, Haifa, Israel. 10.3182/20090616-3-IL-2002.00035 . hal-00387401

\section{HAL Id: hal-00387401 https://hal.science/hal-00387401}

Submitted on 17 Jun 2010

HAL is a multi-disciplinary open access archive for the deposit and dissemination of scientific research documents, whether they are published or not. The documents may come from teaching and research institutions in France or abroad, or from public or private research centers.
L'archive ouverte pluridisciplinaire HAL, est destinée au dépôt et à la diffusion de documents scientifiques de niveau recherche, publiés ou non, émanant des établissements d'enseignement et de recherche français ou étrangers, des laboratoires publics ou privés. 


\title{
Memory Resilient Gain-scheduled State-Feedback Control of LPV Time-Delay Systems with Time-Varying Delays
}

\author{
C. Briat* O. Sename* J.F. Lafay** \\ * GIPSA-Lab, Departement of Control Systems (former LAG), \\ Grenoble Universités, ENSE $E^{3}$ - BP46, 38402 Saint Martin d'Hères - \\ Cedex FRANCE, corentin@briat.info, olivier.sename@gipsa-lab.inpg.fr \\ ** IRCCyN - Centrale de Nantes, 1 rue de la Nö̈ - BP 92101, 44321 \\ Nantes Cedex 3 - FRANCE, Jean-Francois.Lafay@irccyn.ec-nantes.fr
}

\begin{abstract}
This paper is concerned with the stabilization of LTI/LPV time delay systems with time varying delays using LTI and LPV state-feedback controllers. First, a stability test with guaranteed input/output $\mathcal{L}_{2}$ performance is provided in terms of parameter dependent LMIs. The problem of designing both instantaneous and exact-memory state-feedback control laws is solved. The results are then extended to provide memory-resilient controller synthesis conditions. Such controllers are guaranteed to stabilize the considered system even in presence of uncertainty of the delay implemented into the controller. The interest of the approach is finally illustrated through several examples.
\end{abstract}

Keywords: Linear parameter varying systems; Time delay systems; $\mathcal{H}_{\infty}$ gain scheduled controller; Relaxation ; Parameter-dependent LMIs

\section{INTRODUCTION}

Since several years, time-delay systems [Niculescu, 2001, Zhang et al., 2001, Gu et al., 2003, Han, 2005, Gouaisbaut and Peaucelle, 2006] have attracted more and more interest. Indeed, such systems arise in various problems [Niculescu, 2001] such as chemical processes, biological systems, economic systems, and so on... Moreover, the presence of delays in the equations describing the process is often responsible of destabilizing effects and performances deterioration. Indeed, in high-speed systems, even a small time-delay may have a very harmful effect, and thus cannot be neglected. This has motivated the development of many types of stability tests and matched controller design techniques [Xu et al., 2006, Briat et al., 2007, 2008a]. Since the advent of networks and Network Controlled Systems (NCS) appeared the necessity of studying systems with time-varying delays which are a consequence of the propagation of the information through the network.

On the other hand, over the past recent years, LPV systems [Packard, 1994] have been heavily studied since they offer a general way to model and control complex systems such as nonlinear and LTV systems. This fresh upsurge of gain-scheduling based techniques is mainly due to the emergence of LMIs, which provide a powerful formalism for the expression of solutions of many problems arising in systems and control theory. It is important to note that many open problems in LPV framework remain and major improvements are expected as well for stability analysis as for control synthesis.

The stability analysis of LPV time-delay systems is still an open problem and is discussed for instance in [Zhang et al., 2002]. The control of LPV time-delay systems have also been studied in [Wu and Grigoriadis, 2001, Zhang and Grigoriadis, 2005, Briat et al., 2007, 2008b] but this topic has not been extensively studied yet. These systems belong to the intersection of two families of systems and hence inherit from the difficulties of each one. Moreover, additional difficulties arise, for instance several robust control tools which are used to deal with LPV systems (such as projection lemma, dualization lemma [Scherer and Weiland, 2005]) are difficult to apply to LPV timedelay systems. This is mainly due to the use of LyapunovKrasovskii functionals which introduce a larger number of decision matrices compared to Lyapunov functions involved in the analysis of LPV systems.

The aim of the current paper is to find a control law based on a parameter dependent state-feedback of the form

$$
u(t)=K_{0}(\rho) x(t)+K_{h}(\rho) x(t-d(t))
$$

where the gains $K_{0}(\rho)$ and $K_{h}(\rho)$ are aimed to be determined such that the controller stabilizes system

$$
\begin{aligned}
\dot{x}(t)= & A(\rho) x(t)+A_{h}(\rho) x_{h}(t)+B(\rho) u(t) \\
& +E(\rho) w(t) \\
z(t)= & C(\rho) x(t)+C_{h}(\rho) x_{h}(t)+D(\rho) u(t) \\
& +F(\rho) w(t) \\
x(\eta)= & \phi(\eta), \eta \in\left[-h_{M}, 0\right]
\end{aligned}
$$

where $x \in \mathbb{R}^{n}$ is the system state, $x_{h}(t)=x(t-h(t)) \in \mathbb{R}^{n}$ is the delayed state, $u \in \mathbb{R}^{m}$ is the control input, $w \in \mathbb{R}^{p}$ is the exogenous input, $z \in \mathbb{R}^{q}$ is the controlled output and $\phi(\cdot)$ is the functional initial condition.

The delay is assumed to belong to the set

$$
\mathscr{H}:=\left\{h: \mathbb{R}_{+} \rightarrow\left[0, h_{M}\right],|\dot{h}| \leq \mu<1\right\}
$$


with $h_{M}<+\infty$. Note that the delay $d(t)$ involved in the control law is allowed to be different from the system delay $h(t)$. In the first place, the case $d(t)=h(t)$ will be considered and the more general case $d(t)=h(t)+\eta(t)$ with $|\eta(t)| \leq \delta$ will be solved. To this aim, the following set is introduced

$$
\mathscr{D}_{\delta}:=\left\{d: \mathbb{R}_{+} \rightarrow\left[0, h_{M}\right],|d(t)-h(t)| \leq \delta, h \in \mathscr{H}\right\}
$$

and defines the set of controller delay. The derivative of the implemented delay is not considered since it is assumed here that may be very noisy (nondifferentiable). However, if the implemented delay is chosen to be constant (i.e. $d(t)=h_{M} / 2$ which is the value corresponding to a minimal $\delta$ ) then it would be less conservative to take into account the derivative of $d(t)$ which is zero. In such a case, the derivative of the error $\eta(t)$ satisfies $|\dot{\eta}(t)| \leq \mu<1$. This case is not considered in the present paper.

Definition 1.1. We defined here the terminology used to refer to the controllers:

- If $K_{h}(\cdot)=0$ the controller is called 'memoryless controller';

- If $d(t)=h(t)$ for all $t \geq 0$ (i.e. $\delta=0$ in (4)) then the controller is called 'exact memory controller';

- If $|d(t)-h(t)| \leq \delta$ for some $\delta>0$ then the controller is called ' $\delta$-memory resilient controller'.

The set $\mathscr{D}_{\delta}$ is parametrized by the uncertainty radius $\delta>0$. Note that when $\delta=0$ then we have $d(t)=h(t)$ for all $t \geq 0$ and hence the $\delta$-memory resilient controller coincides with exact memory controller. Note also that if $\delta=h_{M}$ then the implemented delay may take any value into $\left[0, h_{M}\right]$ whatever the value of $h(t)$. Hence it could be considered that $h(t)$ is actually unknown, resulting then in the particular case where the memoryless controller and the $h_{M}$-memory resilient controller are actually quite near, structurally speaking. In the examples, it will be illustrated that $\delta$-memory resilient controllers connect together the well-known memoryless and exact-memory controllers by providing a unique and generalized version of the controllers.

The vector of parameters belongs to

$$
\left.\mathscr{P}:=\left\{\rho: \mathbb{R}_{+} \rightarrow U_{\rho} \subset \mathbb{R}^{N_{p}}\right), \dot{\rho} \in \operatorname{co}\left\{U_{\nu}\right\}\right\}
$$

where $N_{p}>0$ is the number of parameters, $U_{\rho}$ is a connected compact set of $\mathbb{R}^{N_{p}}$. $U_{\nu}$ is the set of vertices of the convex set (orthotope) in which the derivative of the parameters evolve and is defined by

$$
U_{\nu}:=\times_{i=1}^{N_{p}}\left\{\underline{\nu}_{i}, \bar{\nu}_{i}\right\}
$$

where $\underline{\nu}_{i}$ and $\bar{\nu}_{i}$ denote respectively the upper and lower bound of $\dot{\rho}_{i}$.

\subsection{Objectives}

The present paper aims at providing solutions to the following problems:

Problem 1.1. Find a parameter dependent state-feedback (1) with $d(t)=h(t)$ which

(a) Asymptotically stabilizes system (2) with or without uncertainties: $x(t) \rightarrow 0$ as $t \rightarrow+\infty$ with $w(t)=0$, for all parameter trajectories $\rho \in \mathscr{P}$ and for all delay $h \in \mathscr{H}$. (b) Provides a guaranteed $\mathcal{L}_{2}$ performance attenuation gain from $w$ to $z$ satisfying $\|z\|_{\mathcal{L}_{2}}<\gamma\|w\|_{\mathcal{L}_{2}}$ with $x(\eta)=0, \eta \in\left[-h_{M}, 0\right], w(t) \neq 0$ for all parameter trajectories $\rho \in \mathscr{P}$ and for all delay $h \in \mathscr{H}$.

Problem 1.2. Find a parameter dependent state-feedback (1) with $d(t) \neq h(t)$ for some $t \geq 0$ which

(a) Asymptotically stabilizes system (2) with or without uncertainties: $x(t) \rightarrow 0$ as $t \rightarrow+\infty$ with $w(t)=0$, for all parameter trajectories $\rho \in \mathscr{P}$, for all delay $h \in \mathscr{H}$ and for all delay $d \in \mathscr{D}_{\delta}$.

(b) Provides a guaranteed $\mathcal{L}_{2}$ performance attenuation gain from $w$ to $z$ satisfying $\|z\|_{\mathcal{L}_{2}}<\gamma\|w\|_{\mathcal{L}_{2}}$ with $x(\eta)=0, \eta \in\left[-h_{M}, 0\right], w(t) \neq 0$ and for all parameter trajectories $\rho \in \mathscr{P}$, for all delay $h \in \mathscr{H}$ and for all delay $d \in \mathscr{D}_{\delta}$.

The first problem is the nominal one where it is assumed that either the delay is unknown or the delay is perfectly known. In the former case, the control law reduces to $u(t)=K_{0}(\rho) x(t)$ while the latter one considers control laws $u(t)=K_{0}(\rho) x(t)+K_{h}(\rho) x(t-h(t))$. However, such a controller is quite difficult to implement due to the strong assumption of exact delay knowledge. Moreover, it is a well known fact that the estimation of delays is a very difficult problem [Belkoura et al., 2008]. Hence, the control law (1) with exact delay value is not acceptable from a practical point of view.

The second problem tries to remedy this problem by allowing for a difference between the values of implemented delay and the delay of the system. Moreover, the stability and $\mathcal{L}_{2}$ input/output performances are guaranteed for variations of the implemented delay within given bounds around the actual delay. This approach makes the implementation of control laws of the form (1) acceptable since, for the first time, resilience with respect to delay uncertainty is provided in terms of LMIs. It is important to note that robustness with respect to delay uncertainty was generally made after the synthesis [Verriest et al., 2002, Sename and Briat, 2006] in LMI based approaches. Moreover the approach provided in this paper can be applied as well for constant delays as for time-varying delays.

The main contributions of the paper are:

- First a delay-dependent stability test for LPV timedelay systems is provided. It is based on the approach of [Han, 2005, Gouaisbaut and Peaucelle, 2006] extended to LPV systems with time-varying delays. Due to the difficulty of deriving LMIs stabilization conditions, a relaxed version is provided and allows for an easy computation of controllers.

- Using the relaxed LMI formulation, constructive solutions to both memoryless and exact-memory statefeedback controller synthesis problems are developed.

- The design of $\delta$-memory resilient controllers is then discussed and a solution based on the scaled-bounded real lemma is provided in terms of LMIs. It is emphasized that this result is the generalization of the classical stabilization results considering exact-memory controllers and memoryless controllers. 
- Finally, the efficiency of the approach is illustrated through several examples and compared to several methods.

For a real square matrix $M$ we define $M^{\mathcal{S}}:=M+M^{T}$. For any matrix $M, M^{T}$ stands for the transpose of $M$. The space of signals with finite energy is denoted by $\mathcal{L}_{2}$ and the energy of $v \in \mathcal{L}_{2}$ is $\|v\|_{\mathcal{L}_{2}}:=\int_{0}^{+\infty} v^{*}(t) v(t) d t$. $\mathbb{S}_{++}^{k}$ denotes the cone of real symmetric positive definite matrices of dimension $k$. $\mathbb{R}_{+}\left(\mathbb{R}_{++}\right)$denotes the set of nonnegative (positive) real numbers. $\star$ denotes symmetric terms in symmetric matrices and in quadratic forms. $\otimes$ denotes the Kronecker product and $\times$ denotes the cartesian product.

The paper is structured as follows: in Section 2 we provide a delay-dependent stability result for LPV timedelay systems with time-varying delays and its associated relaxation. Section 3 is devoted to the development of constructive sufficient conditions to the existence of a parameter dependent state-feedback controllers. Finally, in Section 4, some examples and discussions about the proposed approach are provided.

\section{DELAY-DEPENDENT STABILITY ANALYSIS}

This section is devoted to the stability analysis of LPV time-delay systems of the form (2). The first part concerns the development of a delay-dependent stability result. It is based on the extension of [Han, 2005, Gouaisbaut and Peaucelle, 2006] to LPV time-delay systems with timevarying delays. The immediate LMI conditions are not well suited for synthesis problems due to structural properties such as multiple products between system matrices and decision variables. Hence a relaxed version of the LMI is provided instead. It makes the derivation of stabilization result easier and overall more efficient than approaches based on relaxations made after substitution of the closedloop system matrices into the LMI.

Theorem 2.1. System (2) with no control input (ie. $u(t)=$ $0)$ is asymptotically stable for all $(h, \rho) \in \mathscr{H} \times \mathscr{P}$ and if there exist a continuously differentiable matrix function $P: U_{\rho} \rightarrow \mathbb{S}_{++}^{n}$, a matrix function $X: U_{\rho} \rightarrow \mathbb{R}^{n \times n}$, constant matrices $Q, R \in \mathbb{S}_{++}^{n}$ and a scalar $\gamma>0$ such that the parameter dependent LMI

$$
\left[\begin{array}{ccccccc}
\Phi_{11} & \Phi_{12} & \Phi_{13} & \Phi_{14} & 0 & X(\rho)^{T} & h_{M} R \\
\star & \Phi_{22} & R & 0 & C(\rho)^{T} & 0 & 0 \\
\star & \star & \Phi_{33} & 0 & C_{h}(\rho)^{T} & 0 & 0 \\
\star & \star & \star & -\gamma I_{p} & F(\rho)^{T} & 0 & 0 \\
\star & \star & \star & \star & -\gamma I_{q} & 0 & 0 \\
\star & \star & \star & \star & \star & -P(\rho) & -h_{M} R \\
\star & \star & \star & \star & \star & \star & -R
\end{array}\right] \prec 0
$$

holds for all $(\rho, \nu) \in U_{\rho} \times U_{\nu}$ with

$$
\begin{aligned}
& \Phi_{22}=\frac{\partial P}{\partial \rho}(\rho) \nu-P(\rho)+Q-R \\
& \Phi_{11}=-\left(X(\rho)+X(\rho)^{T}\right) \quad \Phi_{12}=P(\rho)+X(\rho)^{T} A(\rho) \\
& \Phi_{13}=X(\rho)^{T} A_{h}(\rho) \quad \Phi_{14}=X(\rho)^{T} E(\rho) \\
& \Phi_{33}=-(1-\mu) Q-R
\end{aligned}
$$

Moreover the system satisfies $\|z\|_{\mathcal{L}_{2}}<\gamma\|w\|_{\mathcal{L}_{2}}$ for all $(\rho, h) \in \mathscr{P} \times \mathscr{H}$.

Proof : The proof is similar as of [Han, 2005, Gouaisbaut and Peaucelle, 2006] and is based on the LyapunovKrasovskii functional

$$
\begin{aligned}
V= & x^{T}(t) P(\rho) x(t)+\int_{t-h(t)}^{t} x^{T}(\theta) Q x(\theta) d \theta \\
& +\int_{-h_{M}}^{0} \int_{t+\theta}^{t} \dot{x}^{T}(\eta) R \dot{x}(\eta) d \eta d \theta
\end{aligned}
$$

The final LMI can be obtained using the creation lemma (or equivalently the projection lemma [Apkarian et al., 1995].

LMI (7) has the properties that were expected: no multiple products and few increase of conservatism. The removal of multiple products has been allowed through the introduction of a 'slack' variable $X(\rho)$. Concerning the increase of conservatism, it is very difficult to conclude on the 'amount of increase' simply looking at the LMI conditions. The increase of conservatism is induced by the fact that the procedure to obtain (7) introduces a restriction on the set of possible values for $P, Q$ and $R$. This additional conservatism and the (slight) increase of the computational complexity are the price to pay to get easily tractable conditions for the stabilization problem.

\section{DELAY-DEPENDENT STABILIZATION BY STATE-FEEDBACK}

This section is devoted to the control of time-delay systems of the form (2) with a state-feedback controller of general form (1). When the delay is unknown, the particular case of controller with $K_{h}(\cdot)=0$ will be considered and whenever it is exactly known then the delay of the controller $d(t)$ will be considered as identical to $h(t)$. However, due to practical difficulties to have a thorough knowledge of the delay value, the case $d(t)=h(t)+\eta(t)$ with $|\eta(t)| \leq \delta$ is more relevant in practice and will be addressed in the second part of the section. Finally, each result will be extended to consider systems with polytopic type uncertainties.

\subsection{Stabilization using Memoryless and Exact-Memory State-Feedback Controllers}

In this part, control laws of the form

$$
u(t)=K_{0}(\rho) x(t)+K_{h}(\rho) x(t-h(t))
$$

aim to be designed such that Problem 1.1 is solved.

The closed loop system obtained from the interconnection of system (2) and controller (8) is given by

$$
\begin{aligned}
& \dot{x}(t)=A_{c l}(\rho) x(t)+A_{h c l}(\rho) x_{h}(t)+E(\rho) w(t) \\
& z(t)=C_{c l}(\rho) x(t)+C_{h c l}(\rho) x_{h}(t)+F(\rho) w(t)
\end{aligned}
$$

with $A_{c l}(\rho)=A(\rho)+B(\rho) K_{0}(\rho), C_{c l}(\rho)=C(\rho)+$ $D(\rho) K_{0}(\rho), A_{h c l}(\rho)=A_{h}(\rho)+B(\rho) K_{h}(\rho)$ and $C_{h c l}(\rho)=$ $C_{h}(\rho)+D(\rho) K_{h}(\rho)$. The following theorem on nominal stabilization is obtained:

Theorem 3.1. There exists a parameter dependent statefeedback control of the form (8) which asymptotically stabilizes system $(2)$ for all $(h, \rho) \in \mathscr{H} \times \mathscr{P}$ if there exist a continuously differentiable matrix function $P: U_{\rho} \rightarrow$ $\mathbb{S}_{++}^{n}$, constant matrices $Q, R \in \mathbb{S}_{++}^{n}, X \in \mathbb{R}^{n \times n}$, matrix functions $Y_{0}, Y_{h}: U_{\rho} \rightarrow \mathbb{R}^{m \times n}$ and a scalar $\gamma>0$ such that the parameter dependent LMI 


$$
\left[\begin{array}{ccccccc}
\Xi_{11} & \Xi_{12} & \Xi_{13} & E(\rho) & 0 & X & h_{M} R \\
\star & \Xi_{22} & R & 0 & \Xi_{24} & 0 & 0 \\
\star & \star & \Xi_{33} & 0 & \Xi_{34} & 0 & 0 \\
\star & \star & \star & -\gamma I_{p} & F(\rho)^{T} & 0 & 0 \\
\star & \star & \star & \star & -\gamma I_{q} & 0 & 0 \\
\star & \star & \star & \star & \star & -P(\rho) & -h_{M} R \\
\star & \star & \star & \star & \star & \star & -R
\end{array}\right] \prec 0
$$

holds for all $(\rho, \nu) \in U_{\rho} \times U_{\nu}$ where

$$
\begin{aligned}
& \Xi_{11}=-\left(X+X^{T}\right) \\
& \Xi_{12}=P(\rho)+A(\rho) X+B(\rho) Y_{0}(\rho) \\
& \Xi_{23}=A_{h}(\rho) X+B(\rho) Y_{h}(\rho) \\
& \Xi_{22}=\frac{\partial P(\rho)}{\partial \rho} \nu-P(\rho)+Q-R \\
& \Xi_{33}=-(1-\mu) Q-R \\
& \Xi_{24}=\left[C(\rho) X+D(\rho) Y_{0}(\rho)\right]^{T} \\
& \Xi_{34}=\left[C_{h}(\rho) X+D(\rho) Y_{h}(\rho)\right]^{T}
\end{aligned}
$$

Moreover, a suitable control law is given by (8) with gains $K_{0}(\rho)=Y_{0}(\rho) X^{-1}$ and $K_{h}(\rho)=Y_{h}(\rho) X^{-1}$ and the closed-loop system satisfies $\|z\|_{\mathcal{L}_{2}}<\gamma\|w\| \|_{\mathcal{L}_{2}}$ for all $(h, \rho) \in \mathscr{H} \times \mathscr{P}$.

Proof : Substitute the closed-loop system (9) into inequality (7) and set $X$ to be a constant matrix. Then performing a congruence transformation with respect to matrix

$$
\operatorname{diag}\left(I_{3} \otimes X^{-1}, I_{p+q}, I_{2} \otimes X^{-1}\right)
$$

and applying the following linearizing change of variable

$$
\begin{array}{lll}
X \leftarrow X^{-1} & P \leftarrow X^{-T} P X^{-1} & Y_{0} \leftarrow K_{0} X^{-1} \\
Q \leftarrow X^{-T} Q X^{-1} & R \leftarrow X^{-T} R X^{-1} & Y_{h} \leftarrow K_{h} X^{-1}
\end{array}
$$

yields LMI (10). This concludes the proof.

It is important to mention that this result provides a solution to both memoryless and with memory state-feedback controllers. Memoryless controller can be designed by fixing $Y_{h}(\cdot)=0$ in the LMI condition. This solution is preferred when no information is available in real time on the delay value.

On the other hand, it is clear from the expression of the control law (8) and the open-loop system (2) that the exact knowledge of the delay value is crucial for the validity of the control law. Moreover, the estimation or measurement of the delay in a state-delayed system is difficult and hence control law (9) may not be practically valid.

\subsection{Stabilization using $\delta$-Memory Resilient State-Feedback Controllers}

This part completes the results of the latter subsection in which exact knowledge of the delay value is necessary to guarantee the stabilization and the closed-loop performances of the controlled systems (2). In this section, this strong assumption is relieved and a control law of the following form is considered:

$$
u(t)=K_{0}(\rho) x(t)+K_{h}(\rho) x(t-d(t))
$$

where only the approximate value of the delay $d(t) \in \mathscr{D}_{\delta}$ is known. To the authors' knowledge, no such controllers have been studied in the literature using LMI techniques and this study constitutes one the main contributions of this paper.
The closed-loop system given by the interconnection of this control law and system (2) is governed by the expressions

$$
\begin{aligned}
\dot{x}(t)= & A_{c l}(\rho) x(t)+A_{h}(\rho) x_{h}(t)+B(\rho) K_{h}(\rho) x_{d}(t) \\
& +E(\rho) w(t) \\
z(t)= & C_{c l}(\rho) x(t)+C_{h}(\rho) x_{h}(t)+D(\rho) K_{h}(\rho) x_{d}(t) \\
& +F(\rho) w(t)
\end{aligned}
$$

where $x_{h}(t)=x(t-h(t)), x_{d}(t)=x(t-d(t)), A_{c l}(\rho)=$ $A(\rho)+B(\rho) K_{0}(\rho)$ and $C_{c l}(\rho)=C(\rho)+D(\rho) K_{0}(\rho)$.

It is important to note that this system is not simply a system with two delays. Indeed, the main particularity lies in the fact that the delays are coupled through an algebraic inequality which constrains the trajectories of $d(t)$ to evolve within a ball centered around the trajectory of $h(t)$. This additional information needs to be taken into account to analyze correctly the stability and the performances of system (12). To this aim, the following dynamic time-varying operator is introduced:

$$
\Delta\left(z_{0}(t)\right)=\frac{1}{\delta} \int_{t-d(t)}^{t-h(t)} z_{0}(\tau) d \tau
$$

which satisfies $\|\Delta(\xi)\|_{\mathcal{L}_{2}} \leq\|\xi\|_{\mathcal{L}_{2}}$ for every $\xi \in \mathcal{L}_{2}[\mathrm{Gu}$ et al., 2003]. This operator allows to turn system (12) into a one-delay system (i.e. $d(t))$ interconnected with normbounded uncertainty $($ i.e. $\Delta(\cdot))$ :

$$
\begin{aligned}
\dot{x}(t)= & A_{c l}(\rho) x(t)+A_{h c l}(\rho) x_{d}(t)+E(\rho) w(t) \\
& +\delta A_{h}(\rho) w_{0}(t) \\
z(t)= & C_{c l}(\rho) x(t)+C_{h c l}(\rho) x_{d}(t)+F(\rho) w(t) \\
& +\delta C_{h}(\rho) x_{d}(t) \\
z_{0}(t)= & \dot{x}(t) \\
w_{0}(t)= & \Delta\left(z_{0}(t)\right)
\end{aligned}
$$

where $x_{d}(t)=x(t-d(t)), A_{c l}(\rho)=A(\rho)+B(\rho) K_{0}(\rho)$, $A_{h c l}(\rho)=A_{h}(\rho)+B(\rho) K_{h}(\rho), C_{c l}(\rho)=C(\rho)+D(\rho) K_{0}(\rho)$ and $C_{h c l}(\rho)=C_{h}(\rho)+D(\rho) K_{h}(\rho)$.

It is important to note that the uncertainty radius $\delta$ appears explicitly in the expression of the transformed system. Hence (14) is a comparison system for (12) where the delays satisfy $|d(t)-h(t)| \leq \delta$.

Remark 3.1. The use of the operator $\Delta(\cdot)$ introduces additional dynamics in system (14) as shown in [Gu et al., 2003] and hence system (14) is not equivalent to system (12). The analysis of the conservatism is performed for constant time-delay only since the provided approach is based on frequency-domain methods. An important result states that the conservatism only depends on the eigenvalues of the matrix $A_{h}$ and if the matrix $A_{h}$ is Hurwitz then both systems are equivalent. In the stabilization problem, the matrix acting on the delayed state is 'controlled': $A_{h}+$ $B K_{h}$ and hence can be made Hurwitz by an appropriate choice of the matrix $K_{h}$ (assuming that the pair $\left(A_{h}, B\right)$ is stabilizable). Hence, the impact of additional dynamics may be less critical in the stabilization problem.

The following theorem provides a solution to the Problem 1.2 which considers the synthesis of $\delta$-memory resilient controllers:

Theorem 3.2. There exists a parameter dependent statefeedback control law of the form (11) which asymptotically stabilizes system $(2)$ for all $(h, d, \rho) \in \mathscr{H} \times \mathscr{D}_{\delta} \times \mathscr{P}$ if there exist a continuously differentiable matrix function $P: U_{\rho} \rightarrow \mathbb{S}_{++}^{n}$, matrices $Q, R \in \mathbb{S}_{++}^{n}, X \in \mathbb{R}^{n \times n}$, 
$Y_{0}, Y_{h}: U_{\rho} \rightarrow \mathbb{R}^{m \times n}$ and a scalar $\gamma>0$ such that the parameter dependent LMI

$$
\left[\begin{array}{cc}
\Omega_{1} & \Omega_{2} \\
\star & \Omega_{3}
\end{array}\right] \prec 0
$$

holds for all $(\rho, \nu) \in U_{\rho} \times U_{\nu}$ and where

$\begin{aligned} \Omega_{1}= & {\left[\begin{array}{cccccc}\Omega_{11} & \Omega_{12} & \Omega_{13} & \Omega_{14} & E(\rho) & 0 \\ \star & \Omega_{22} & R & \delta R & 0 & \Omega_{26} \\ \star & \star & \Omega_{33} & \delta \Omega_{33} & 0 & \Omega_{36} \\ \star & \star & \star & \Omega_{44} & 0 & \delta C_{h}(\rho)^{T} \\ \star & \star & \star & \star & -\gamma I_{p} & F^{T} \\ \star & \star & \star & \star & \star & -\gamma I_{q}\end{array}\right] } \\ \Omega_{2}= & {\left[\begin{array}{cccccc}X^{T} & 0 & 0 & 0 & 0 & 0 \\ S(\rho) & 0 & 0 & 0 & 0 & 0 \\ h_{M} R & 0 & 0 & 0 & 0 & 0\end{array}\right]^{T} \quad \Omega_{3}=\left[\begin{array}{ccc}-P(\rho) & -S(\rho) & -h_{M} R \\ \star & -S(\rho) & 0 \\ \star & \star & -R\end{array}\right] }\end{aligned}$

$\Omega_{11}=-\left(X+X^{T}\right) \quad \Omega_{12}=P(\rho)+A(\rho) X+B(\rho) Y_{0}(\rho)$

$\Omega_{14}=\delta A_{h}(\rho) X \quad \Omega_{13}=A_{h}(\rho) X+B(\rho) Y_{h}(\rho)$

$\Omega_{33}=-(1-\mu) Q-R \quad \Omega_{22}=\frac{\partial P}{\partial \rho} \nu-P(\rho)+Q-R$

$\Omega_{44}=\delta^{2} \Omega_{33}-S(\rho) \quad \Omega_{36}=\left(C_{h}(\rho) X+D(\rho) Y_{h}(\rho)\right)^{T}$

$\Omega_{26}=\left(C(\rho) X+D(\rho) Y_{0}(\rho)\right)^{T}$

In such a case, the controller gains can be computed using the relations $K_{0}(\rho)=Y_{0}(\rho) X^{-1}$ and $K_{h}(\rho)=Y_{h}(\rho) X^{-1}$.

Moreover the closed-loop system satisfies $\|z\|_{\mathcal{L}_{2}}<\gamma\|w\|_{\mathcal{L}_{2}}$ for all $(\rho, h, d) \in \mathscr{P} \times \mathscr{H} \times \mathscr{D}_{\delta}$.

Proof : The proof is only sketched for brevity. It is based on a simple application of the scaled-small gain with the same relaxation procedure as for the obtention of Theorem 3.1 .

Remark 3.2. It is important to note that when $\delta$ tends to 0, then LMI (15) tends to LMI (10) provided that the matrix $S(\rho) \succ 0$ is chosen sufficiently small. This means that Theorem 3.2 is a direct generalization of Theorem 3.1.

\section{EXAMPLE}

This section is devoted to examples and discussions about the provided approach. It will be illustrated that the current approach improves result of the literature in the control of LPV time-delay systems. Moreover, the connection between memoryless and exact-memory controllers made by $\delta$-memory resilient controllers will be illustrated.

\subsection{Example 1: Instantaneous state-feedback}

Let us consider system (2) borrowed from [Wu and Grigoriadis, 2001] and modified in [Zhang and Grigoriadis, 2005]

$$
\begin{aligned}
\dot{x}(t)= & {\left[\begin{array}{cc}
0 & 1+\phi \sin (t) \\
-2 & -3+\delta \sin (t)
\end{array}\right] x(t)+\left[\begin{array}{c}
\phi \sin (t) \\
0.1+\delta \sin (t)
\end{array}\right] u(t) } \\
& +\left[\begin{array}{cc}
\phi \sin (t) & 0.1 \\
-0.2+\delta \sin (t) & -0.3
\end{array}\right] x_{h}(t)+\left[\begin{array}{l}
0.2 \\
0.2
\end{array}\right] w(t) \\
z(t)= & {\left[\begin{array}{cc}
0 & 10 \\
0 & 0
\end{array}\right] x(t)+\left[\begin{array}{c}
0 \\
0.1
\end{array}\right] u(t) }
\end{aligned}
$$

where $x_{h}(t)=x(t-h(t)), \rho(t)=\sin (t), \phi=0.2$ and $\delta=0.1$.

Choosing as in [Zhang and Grigoriadis, 2005], $h_{M}=0.5$ and $\mu=0.5$, a memoryless control law is computed using

\begin{tabular}{c|c}
$\mu$ & $h_{M}$ \\
\hline \hline 0 & 929.1372 \\
0.5 & 371.0928 \\
0.9 & 6.9218 \\
0.99 & 2.9325
\end{tabular}

Table 1. Evolution of the delay margin $h_{M}$ with respect to the bound on the delay derivative $\mu$ such that $\gamma<10$

Theorem 3.1. This yields a minimal value $\gamma^{*}=1.9089$ which is better than all results obtained before: in [Zhang and Grigoriadis, 2005], a minimal value of $\gamma=3.09$ is found while in Briat et al. [2008b] an optimal value of $\gamma=2.27$ is obtained (using a nonlinear approach). The corresponding controller is given by

$$
K_{0}(\rho)=\left[\begin{array}{l}
-1.0535-2.9459 \rho+1.9889 \rho^{2} \\
-1.1378-2.6403 \rho+1.9260 \rho^{2}
\end{array}\right]^{T}
$$

Note that better results are obtained while the controller has smaller coefficients than in the other approaches. It is hence expected to have a smaller control input which should remain within acceptable bounds, even in presence of disturbances. This is another benefit of the proposed approach.

The influence of $\mu$ on the delay-margin (with $\mathcal{L}_{2}$ performance constraint) is detailed on Table 4.1 where, as expected, the delay margin decreases as the value of $\mu$ increases. Moreover, the results of [Zhang and Grigoriadis, 2005] are more conservative than those of Table 4.1 since the delay-margin is always smaller than 1.4 (for $\gamma<10$ ) whatever the value of $\mu$ (see Figure 1. in [Zhang and Grigoriadis, 2005]).

As a final remark, the value of $h_{M}$ obtained for $\mu=$ 0 is large and this suggests that the system is delayindependent stabilizable for this specific value of $\mu$.

\subsection{Example 2: Delayed-State Feedback}

Still considering system (16) but with $h_{M}=10$ and $\mu=0.9$, we look for an instantaneous state-feedback controller of the form $u(t)=K_{0}(\rho) x(t)$ and Theorem 3.1 yields:

$$
K_{0}(\rho)=\left[\begin{array}{c}
0.5724-6.3679 \rho-1.4898 \rho^{2} \\
-0.7141-4.1617 \rho-0.8425 \rho^{2}
\end{array}\right]^{T}
$$

which ensures a closed-loop $\mathcal{L}_{2}$ input/output performance level of 12.8799. Now an exact-memory state-feedback control law $u(t)=K_{0}(\rho) x(t)+K_{h}(\rho) x(t-h(t))$ is computed and the following gains are given by

$$
\begin{aligned}
& K_{0}(\rho)=\left[\begin{array}{c}
1.0524-2.8794 \rho-0.4854 \rho^{2} \\
-0.7731-1.8859 \rho+0.1181 \rho^{2}
\end{array}\right]^{T} \\
& K_{h}(\rho)=\left[\begin{array}{l}
-0.6909+0.5811 \rho+0.1122 \rho^{2} \\
-0.0835+0.3153 \rho+0.0689 \rho^{2}
\end{array}\right]^{T}
\end{aligned}
$$

which ensures a closed-loop $\mathcal{L}_{2}$ performance level 4.1641 . The gain of performances resulting from the use of a controller involving a delayed term is evident.

It is aimed now at finding a $\delta$-memory resilient statefeedback controller. Using Theorem 3.2, the achieved minimal closed-loop $\mathcal{L}_{2}$ performance with respect to $\delta$ is plotted in Figure 1. As expected the minimal $\mathcal{L}_{2}$ performance level grows whenever the delay uncertainty radius grows. 


\begin{tabular}{l|l||l|l} 
Exact memory & $\gamma=4.1641$ & Memoryless & $\gamma=12.8799$ \\
\hline 0-resilient & $\gamma=4.1658$ & 10-resilient & $\gamma=12.7167$
\end{tabular}

Table 2. Comparison of the results obtained using Theorem 3.1 and Theorem 3.2

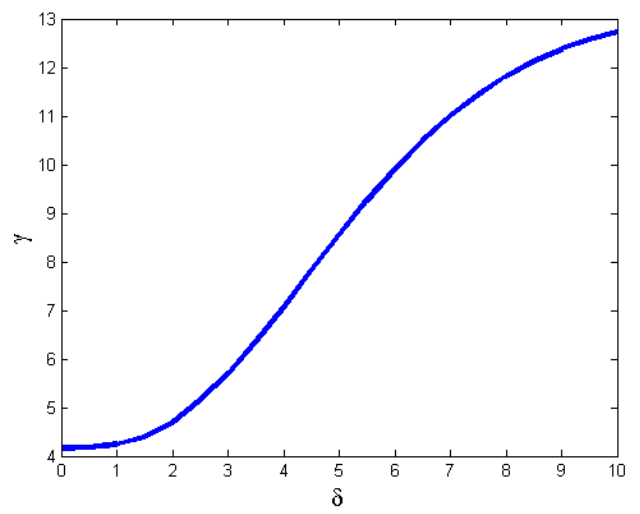

Fig. 1. Evolution of the worst-case $\mathcal{L}_{2}$ gain of the closedloop system with respect to maximal delay uncertainty $\delta$

This means that, the achieved closed-loop performances are deteriorated when the delay is badly known.

Moreover, there are two remarkable values for the worstcase $\mathcal{L}_{2}$ gain, respectively obtained for $\delta=0$ and $\delta=h_{M}$. For these particular values we have:

$$
\begin{aligned}
& \left.\gamma\right|_{\delta=0}=4.1658 \\
& \left.\gamma\right|_{\delta=10}=12.7167
\end{aligned}
$$

and we retrieve the preceding results. When the delay is exactly known (i.e. $\delta=0$ ), the $\mathcal{L}_{2}$ performance index and the controller are very close (quite identical) to the results obtained using Theorem 3.1 which considers exactmemory controllers. This illustrates well Remark 3.2.

This illustrates that $\delta$-memory resilient controllers define the intermediary behavior of the closed-loop system between the two extremal controllers: the memoryless and the exact-memory controllers. The emphasis of this continuity between memoryless and exact memory controller through $\delta$-memory resilient controllers constitutes one of the main contribution of the approach.

\section{CONCLUSION}

The current paper introduces a new approach to the stabilization of LPV time-delay systems using parameter dependent state-feedback controllers. First, a delaydependent stability test with $\mathcal{L}_{2}$ performance analysis for LPV time-delay systems with time-varying delays is provided in terms of parameter dependent LMIs.

Stabilization results are then provided and allow to compute both memoryless and exact-memory controllers. However, due to the difficulty of estimating delays, the latter controllers are generally non-implementable in practice. This has motivated the second result where the uncertainty on the implemented delay is taken into account and the stability $/ \mathcal{L}_{2}$ performance are guaranteed even in presence of these uncertainties. This innovative part is the main contribution of the paper and the examples show the effectiveness of the approach. It turns out that $\delta$ resilient memory controllers connects together memoryless and exact-memory controllers. There is a continuous path between memoryless and exact-memory controllers and this path is parametrized by the delay uncertainty radius $\delta$ involved in the definition of $\delta$-resilient memory controllers as illustrated in the examples.

\section{REFERENCES}

P. Apkarian, P. Gahinet, and G. Becker. Self-scheduled control of linear parameter varying systems: A design example. Automatica, 31(9):1251-1261, 1995.

L. Belkoura, J.P. Richard, and M. Fliess. A convolution approach for delay systems identification. In IFAC World Congress, Seoul, Korea, 2008.

C. Briat, O. Sename, and J-F. Lafay. A LFT $/ \mathcal{H}_{\infty}$ statefeedback design for linear parameter varying time delay systems. In European Control Conference 2007, Kos, Greece, 2007.

C. Briat, O. Sename, and J.F. Lafay. Delay-scheduled state-feedback design for time-delay systems with timevarying delays. In IFAC World Congress, Korea, Seoul, 2008a.

C. Briat, O. Sename, and J.F. Lafay. Parameter dependent state-feedback control of LPV time delay systems with time varying delays using a projection approach. In IFAC World Congress, Korea, Seoul, 2008b.

F. Gouaisbaut and D. Peaucelle. Delay dependent robust stability of time delay-systems. In $5^{\text {th }}$ IFAC Symposium on Robust Control Design, Toulouse, France, 2006.

K. Gu, V.L. Kharitonov, and J. Chen. Stability of TimeDelay Systems. Birkhäuser, 2003.

Q.L. Han. Absolute stability of time-delay systems with sector-bounded nonlinearity. Automatica, 41:2171-2176, 2005.

S.-I. Niculescu. Delay effects on stability. A robust control approach, volume 269. Springer-Verlag: Heidelbeg, 2001.

A. Packard. Gain scheduling via linear fractional transformations. Systems and Control Letters, 22:79-92, 1994.

C. Scherer and S. Weiland. Linear Matrix Inequalities in Control. 2005.

O. Sename and C. Briat. Observer-based $\mathcal{H}_{\infty}$ control for time-delay systems: a new LMI solution. In IFAC TDS Conference, L'Aquila, Italy, 2006.

E. I Verriest, O. Sename, and P. Pepe. Robust observercontroller for delay-differential systems. In IEEE Conference on Decision and Control, Las Vegas, USA, 2002.

F. $\mathrm{Wu}$ and K.M. Grigoriadis. LPV systems with parameter-varying time delays: analysis and control. Automatica, 37:221-229, 2001.

S. Xu, J. Lam, and Y. Zhou. New results on delaydependent robust $\mathcal{H}_{\infty}$ control for systems with timevarying delays. Automatica, 42(2):343-348, 2006.

F. Zhang and K.M. Grigoriadis. Delay-dependent stability analysis and $\mathcal{H}_{\infty}$ control for state-delayed LPV system. In Conference on Control and Automation, 2005.

J. Zhang, C.R. Knospe, and P. Tsiotras. Stability of timedelay systems: Equivalence between lyapunov and scaled small-gain conditions. IEEE Transactions on Automatic Control, 46:482-486, 2001.

X. Zhang, P. Tsiotras, and C. Knospe. Stability analysis of LPV time-delayed systems. Int. Journal of Control, 75:538-558, 2002. 